\title{
Mediação parental do uso da internet pelas crianças
}

\author{
Parental mediation of internet use by children \\ Mediación parental del uso de internet por niños
}

\author{
Simone Maidel \\ Mauro Luis Vieira ${ }^{* *}$
}

\begin{abstract}
Resumo
Investigou-se a mediação parental de pais universitários brasileiros $(\mathrm{n}=50)$ para guiar o uso da internet pelos filhos de 7 a 12 anos, partindo da hipótese de que as crenças relacionadas a esse uso interferem no tipo de mediação adotada. Um questionário fechado foi aplicado, e os resultados indicam que os respondentes, em média com 28 anos $( \pm 8.58)$, recorrem a um tipo de mediação mista, caracterizado tanto pela estipulação de regras quanto pela supervisão, conversa e acompanhamento das atividades do filho na internet, embora demonstrem preferência pelo uso da mediação ativa e do uso acompanhado. Verificou-se que pais que mais conversam e orientam também aplicam mais regras para o uso da internet pelos filhos, entretanto as crenças parecem não afetar o tipo de mediação utilizada. Tal resultado precisa ser relativizado, uma vez que se trata de um estudo-piloto, mas aponta para necessidade de mais estudos sobre o tema.
\end{abstract}

Palavras-chave: Crianças. Internet. Mediação parental. Crenças parentais.

\begin{abstract}
We investigated if the main beliefs of parents are related to the type of parental mediation used to guide the use of Internet by children. A questionnaire was applied to 50 parents of both sexes and at least one child aged 7-12 years. The results indicate that respondents, on average 28 years old $( \pm 8.58)$, resort to a mixed type of mediation, characterized by the stipulation of rules for the children's use and the supervision, discussion and monitoring of activities of the child on the Internet, although they prefer using active mediation and co-using. It was found that beliefs do not seem to affect the type of mediation used. This result needs to be relativized,
\end{abstract}

\footnotetext{
Texto recebido em junho 2013 e aprovado para publicação em novembro de 2014.

Doutora em Psicologia e mestra em Neurociências pela Universidade Federal de Santa Catarina (UFSC), psicóloga, integrante do Núcleo de Estudos e Pesquisas sobre Desenvolvimento Infantil (NEPeDI/UFSC) e pesquisadora do grupo de estudo Filosofia da Mente e Ciências Cognitivas (Unidavi). E-mail: simonemaidel@gmail.com.

* Pós-doutor na Dalhousie University em Halifax (Canadá), doutor em Psicologia Experimental pela Universidade de São Paulo, professor do Departamento de Psicologia na UFSC, trabalha na linha de pesquisa sobre cuidados parentais e desenvolvimento infantil no Programa de Pós-Graduação em Psicologia da UFSC, coordenador do Núcleo de Estudo e Pesquisas sobre Desenvolvimento Infantil (NEPeDI/UFSC). E-mail: maurolvieira@gmail.com.

Endereço para correspondência: Núcleo de Estudo e Pesquisas sobre Desenvolvimento Infantil / Departamento de Psicologia. Centro de Filosofia e Ciências Humanas/UFSC. Campus Universitário - Trindade. Florianópolis-SC, Brasil. CEP: 88.040-970.
} 
since the sample size is limited and scales used have not been validated, but points to the need for further studies on the subject.

Keywords: Children. Internet. Parental mediation. Parental beliefs.

\section{Resumen}

Se investigó la mediación parental de padres universitarios brasileños $(\mathrm{n}=50)$ para orientar el uso de internet por los hijos de 7 a 12 años, partiendo de la hipótesis de que las creencias relacionadas con este uso interfieren en el tipo de mediación adoptada. Los resultados indican que los encuestados, en promedio con 28 años $( \pm 8,58)$, recurren a un tipo de mediación mixta, que se caracteriza tanto por la estipulación de reglas como por la supervisión, diálogo y acompañamiento de las actividades del hijo en internet, aunque prefieren el uso de la mediación activa y el uso acompañado. Se comprobó que los padres que más dialogan, mientras que las creencias no parecen afectar al tipo de mediación utilizada. Este resultado debe ser relativizado, ya que se trata de un estudio piloto, pero señala la necesidad de realizar más estudios sobre el tema.

Palabras clave: Niños. Internet. Mediación parental. Creencias parentales.

\section{Introdução}

0

crescente acesso que as crianças têm ao universo dos computadores, da internet, celulares e de outras tecnologias digitais transforma consideravelmente o dia a dia delas. Cada vez mais novas, já fazem uso habitual e massivo desses recursos tanto para tarefas escolares e pesquisas quanto para comunicação e, sobretudo, divertimento. Tanto positiva quanto negativamente, esse uso excessivo tem sido associado a reflexos diretos e indiretos no comportamento das crianças ou mesmo em seu desenvolvimento.

O uso frequente, por exemplo, dos computadores, jogos eletrônicos e da internet pelas crianças pode facilitar diferentes tipos de aprendizado (Swing, Gentile \& Andersen, 2008), pode melhorar habilidades de leitura de crianças com um baixo desempenho inicial (Jackson, Eye, Witt, Zhao \& Fitzgerald, 2011), pode despertar habilidades como aceder, avaliar e usar a informação de forma eficiente (Ponte \& Vieira, 2008), pode estimular habilidades para o processamento e seleção de informações, tomada de decisão, estabelecimento de estratégias de solução de problemas (Mungunba, Valdés, Matos \& Silva, 2003; Wilson et al., 2009). Por outro lado, o uso demasiado dessas mídias digitais também pode causar problemas físicos, sociais e psicológicos (Çankayaa \& Odaba i, 2009), 
como desencadear comportamentos de dependência (Chang \& Law, 2008), de depressão (Morrison \& Gore, 2010), de ansiedade e estresse (Mendes, 2011) e até de transtorno de déficit de atenção e hiperatividade (Rodríguez \& Morales, 2007). Também pode, ainda, aumentar o risco de obesidade e tendinite (Subrahmanyam, Kraut, Greenfield \& Gross, 2000), levar ao desenvolvimento de comportamentos e pensamentos agressivos (Alves, 2005; Gentile \& Stone, 2005; Swing et al., 2008), e até gerar dificuldades, especialmente aos mais novos, para entender as fronteiras entre a vida real e a virtual (Turkle, 1995). Uma série de prejuízos na saúde observados em crianças e adolescentes americanos com idades entre 2 e 18 anos, como insônia, agitação, obesidade, problemas de visão, etc., também são apontados pela Academia Americana de Pediatria como decorrentes do uso diário de tais recursos por períodos que excedem quatro horas diárias - considerado um uso demasiado por ultrapassar, em muito, o tempo que as crianças gastam envolvidas com qualquer outra atividade (Barkin et al., 2006).

Vistas algumas das implicações apontadas para o uso de tais mídias pelas crianças, o papel dos pais na gestão desse processo pode ser considerado central, especialmente porque a casa da família é apontada como o lugar mais comum para esse uso, especialmente a internet (Comitê Gestor da Internet Brasil [CGI], 2011; Tabone \& Messina, 2010; Almeida et al., 2011; Valcke, Bonte, De Wever $\&$ Rots, 2010).

Além de oportunizar o acesso das crianças ao uso das mídias (televisão, videojogo, telemóvel, internet, computador, etc.), os pais também são os grandes responsáveis pelo gerenciamento e educação dos filhos para tal utilização, o que inclui a elaboração de normas, restrições, orientações e táticas sociais, bem como de supervisão ou monitoramento.

Ainda que exista variação quanto à terminologia empregada na literatura para designar as estratégias parentais direcionadas às mídias, a noção de "mediação" tem prevalecido, como se observa em vários estudos desde a década de 1990 até a atualidade, como os de Austin (1993), Valkenburg, Krcmar, Peeters e Marseille (1999), Nathanson (2001), Warren (2005), Livingstone e Helsper (2008), Çankaya e Odaba i (2009) e de Almeida, Alves e Delicado (2011). De modo geral, nesse contexto, entende-se a mediação parental como um processo pelo qual os pais (pai/mãe) influenciam, com suas condutas, valorizações e verbalizações nas modalidades de uso e significações que os filhos têm a respeito das mídias. Assim, o termo "mediação" utilizado tanto é capaz de expressar adequadamente a gestão parental presente na relação entre a criança e a mídia como amplifica o papel parental ao considerar, além das restriçôes, as estratégias sociais e interpretativas tão bem quanto as atividades de monitoramento por eles empregadas (Mondin, 2008). 
Comumente, são relatados na literatura três tipos principais de mediação parental para o uso de mídias pelas crianças: a mediação ativa (MA), a mediação restritiva (MR) e a mediação "uso acompanhado" (UA). ${ }^{1}$ A primeira, MA, designa um tipo de mediação na qual há conversa, orientação ou discussão crítica dos pais com a criança sobre o conteúdo ou atividade, sobretudo enquanto ela ainda está utilizando a mídia. Esse tipo de mediação inclui tanto as formas instrutivas e informativas quanto as críticas, manifestações de desaprovação ou acordos com a criança para o uso. Já a MR refere-se a um tipo de mediação que envolve o estabelecimento e uso de regras explícitas para a utilização (por exemplo, quando a criança pode utilizar, tempo gasto na atividade, local de utilização, conteúdo e atividades permitidas, etc.), mas diferentemente da $\mathrm{MA}$, não inclui qualquer explicação ou conversa com a criança. E, por fim, a mediação do tipo UA consiste na presença do pai, da mãe ou outro responsável, quando a criança está engajada na atividade, seja de maneira deliberada (por exemplo, brincando com a criança) ou casual (por exemplo, ficando perto ou observando o que ela faz); mas como a MR, também não envolve conversa ou orientação da criança nesse momento (Almeida, Alves, Delicado \& Carvalho, 2011; Livingstone \& Helsper, 2008; Livingstone, Haddon, Görzig \& Ólafsson, 2011; Lwin, Stanaland \& Miyazaki, 2008; Mesch, 2009; Pasquier, 2001; Çankaya \& Odaba i, 2009).

Os pais também se utilizam dos recursos tecnológicos e midiáticos para facilitar o desenvolvimento de valores desejados nas crianças (por exemplo, desempenho acadêmico) e para suporte de atividades familiares compartilhadas (por exemplo, construção de interesses comuns, orientação quanto as crenças e valores familiares, acompanhamento das atividades dos filhos) (Fujioka \& Austin, 2002; Nathanson, 2002; Warren, 2005). E assim como o uso da mídia pelas crianças, também o tipo de mediação parental utilizada tem implicações para o comportamento e desenvolvimento das crianças.

Segundo Austin (1993), a MA tanto pode auxiliar a criança a desenvolver seus próprios referenciais de "bom" e "mau" para o conteúdo de mídia como pode levar a criança a questionar e avaliar criticamente tal conteúdo. Mas também pode levar a criança a rejeitar a autoridade parental, principalmente quando os pais discordam de algo que a criança avalia como sendo "bom", por exemplo, em situações em que a criança prefere um jogo on-line de conteúdo violento, apesar da contraindicação parental (Pasquier, 2001). A MR parece dificultar o desenvolvimento de um espírito crítico na criança, pois a ausência de conversa priva a criança de elementos para entender o porquê da regra, além de instigar sua curiosidade "pelo proibido", fazendo-a burlar ou desobedecer às regras dos

\footnotetext{
1 Esse tipo de mediação aparece na literatura originalmente como "coviewing", "social-coviewing" ou "co-using", pois inicialmente o termo referia-se a televisão (Valkenburg et al., 1999). Optou-se pelo termo "uso acompanhado" por considerarmos mais adequado à postura ativa decorrente da interatividade oportunizada pelas novas mídias digitais.
} 
pais quando não está sob vigilância. Mesmo o UA e o simples monitoramento, acompanhamento ou supervisão das atividades das crianças enquanto envolvidas com as mídias, sem a devida orientação, discussão ou críticas por parte dos pais, podem levar a criança ao entendimento de "aprovação" dessas atividades (Kuhlemeier \& Hemler, 2007; Rosen, Cheever \& Carrier, 2008; O’Keeffe, 2010).

Especificamente com relação à internet, autores como Lee e Chae (2007) alertam que ainda são insuficientes os estudos que investigam como os pais regulam, supervisionam ou controlam o uso da internet pelas crianças. Apesar disso, vem crescendo o número de estudos qualitativos e quantitativos que examinam o papel da internet nas famílias e as estratégias parentais para regular o acesso das crianças (Tripp, 2010).

Os resultados do estudo de Barkin et al. (2006), com 1.831 famílias do Canadá e de Porto Rico, por exemplo, indicaram que cerca de $60 \%$ dos pais usam várias estratégias de mediação (UA, MA, MR ou programas bloqueadores, etc.) para regular o uso do computador/internet, televisão e videojogos dos filhos, $23 \%$ se utilizam somente de MR, $11 \%$ orientam ou conversam com as crianças sobre o uso da internet, ou seja, utiliza-se de MA e, ainda, que $6 \%$ dos pais da amostra afirmaram não usar nenhum tipo de mediação, ou seja, deixam a critério dos filhos quando utilizar, a escolha das atividades, o período de tempo dedicado a elas, etc.. Também nos Estados Unidos, Mesch (2009) investigou 935 adolescentes de 12 a 17 anos e um de seus pais, e encontrou que os pais, em sua maioria, impóem regras para o uso da internet, como websites que o filho pode ou não visitar (86\%), tempo que pode permanecer on-line $(66 \%)$ ou então monitoram regularmente os sites visitados pela criança $(65 \%)$ ou usam filtros e regras sobre o tipo de informação que os filhos podem passar na internet (56\%). Resultado semelhante foi encontrado no contexto português, conforme apresentado por Almeida, Alves, Delicado e Carvalho (2011), com base no inquérito realizado com 3.049 crianças (cerca de $60 \%$ afirmaram que os pais impõem regras de utilização aos filhos semelhantes às apontadas por Mesch (2009).

Já no Reino Unido, conforme os resultados do projeto "UK Children Go Online", provenientes de entrevistas com 1.511 crianças de 9 a 17 anos e um de seus pais $(\mathrm{N}=906)$, Livingstone e Bober (2006) verificaram que os pais ingleses acompanham as atividades do filho na internet seja perguntando à criança o que ela faz (81\%), auxiliando-a em suas atividades (57\%), sentando-se ao seu lado enquanto ela navega (31\%) ou mesmo verificando o histórico do computador (41\%). Referente ao mesmo estudo, Livingstone e Helsper (2008) ressaltam que os pais ingleses predominantemente (64\%) conversam com os filhos sobre o uso 
da internet (MA), mas assim como no contexto português e americano, utilizamse de regras para o tempo da criança on-line (53\%), usam filtros bloqueadores $(33 \%)$ e observam as atividades da criança quando está on-line (46\%). Mais especificamente, esses autores apontam que $47 \%$ da amostra se utiliza de uma mediação do tipo mista (MA, MR, UA, etc.).

No Brasil, o Comitê Gestor da Internet Brasil (CGI, 2011), com base em 2.516 entrevistas realizadas com crianças brasileiras de 5 a 9 anos e pelo menos um de seus pais, aponta que a principal mediação utilizada pelos pais é a orientação da criança para o uso da internet $(40 \%)$, seguida pelo controle do tempo que a criança pode permanecer on-line (34\%) e a observação da criança enquanto ela usa a internet (31\%). Porém $21 \%$ dos pais brasileiros declararam não realizar nenhum tipo de restrição ou controle para o uso da internet pelos filhos, um dado preocupante se levar-se em conta a pouca idade das crianças entrevistadas na pesquisa. Aslanidou e Menexes (2008), em um estudo realizado com 418 jovens gregos de 12 a 18 anos, verificaram que, embora $40 \%$ destes jovens tenham reconhecido a existência de algum tipo de supervisão por parte dos pais, mais de $60 \%$ declararam que eles não impõem qualquer regra ou limitação para sua utilização da internet.

Com base na revisão de literatura sobre o tema proposto neste estudo, sabe-se que o tipo de mediação dos pais com relação ao uso da internet pelas crianças sofre influências de fatores como a idade e o gênero da criança bem como do nível de instrução e do próprio conhecimento que têm da internet. E embora alguns estudos (Pijpers \& Pardoen, 2009; Tripp, 2010) apontem que as crenças que os pais têm sobre a influência, positiva ou negativa, que determinada mídia poderá ter sobre o comportamento ou desenvolvimento de seu filho pode influenciar sua mediação, ainda se constata uma lacuna no que diz respeito às crenças parentais relacionadas ao uso da internet pelas crianças.

Além disso, mesmo com a expansão dos estudos observada nessa área, vários pesquisadores (Eastin, Greenberg \& Hofschire, 2006; Kutner, Olson, Warner \& Herzog, 2008; Livingstone \& Bober, 2006; Livingstone \& Helsper, 2008; Pijpers \& Pardoen, 2009; Warren, 2005; Weinberger, Anderson \& Schumacher, 2009) evidenciam a premente necessidade de investigações que envolvam diversos aspectos relacionados aos cuidadores (pais e, ou, mães) e seu papel no uso que as crianças fazem da internet, especialmente pelas potenciais decorrências que esse uso pode trazer ao desenvolvimento infantil.

Buscando contribuir nesse sentido, especialmente no contexto brasileiro, este estudo-piloto tem por objetivo investigar se as crenças de pais universitários brasileiros quanto ao uso da internet pelos filhos (na faixa etária de 7 a 12 anos) 
estão relacionadas ao tipo de mediação parental que utilizam. Adicionalmente, verifica-se a possibilidade de variáveis demográficas interferirem no tipo de mediação parental empregada. Parte-se da hipótese de que crenças relacionadas a aspectos negativos ou positivos do uso da internet pelas crianças poderiam alterar o tipo de mediação parental utilizada, ou seja, crenças relacionadas aos efeitos não desejáveis do uso da internet para os seus filhos estariam relacionadas ao maior uso de controle e regras por parte dos pais; e crenças positivas, à menor utilização destas; bem como se admite a possibilidade de que fatores sociodemográficos influenciem no tipo de mediação utilizada.

\section{Método}

\section{Participantes}

Este estudo foi realizado em uma instituição de ensino superior particular, localizada na Grande Florianópolis-SC (Brasil), com uma amostra voluntária composta por 50 estudantes, 22 do sexo masculino (44\%) e 28 do sexo feminino (56\%), com idades compreendidas entre os 25 e 50 anos $(M=28,19 ; \mathrm{DP}=8,58)$, pertencentes a três turmas do último período do curso de Administração, período noturno, e que tinham filhos com idades entre 7 e 12 anos $(M=10,10$; $\mathrm{DP}=1,58)$.

\section{Procedimento}

Por meio de comunicação on-line (e-mail) enviada pelo coordenador do curso de Administração, todos os alunos matriculados no último período noturno receberam informações sobre a ocorrência desse estudo na instituição e um convite para participar. Além do critério de inclusão estipulado (pelo menos um filho com idade entre 7 e 12 anos), os interessados em participar deveriam preencher e assinar o termo de consentimento livre e esclarecido (TCLE). A recolha de dados foi realizada de maneira coletiva em uma sala de aula, nas dependências da própria instituição, sendo que o preenchimento do questionário autoaplicável durou, em média, 50 minutos.

Os dados obtidos foram inseridos numa planilha e tratados com programa estatístico SPSS, versão 17. Embora seja uma amostra pequena $(n=50)$, a checagem dos dados quanto à normalidade indica que, apesar de desvios aceitáveis da distribuição (curtose e assimetria $< \pm 0,4$ ), entre a nossa distribuição e a distribuição normal, não houve diferença estatística (K-S com $\mathrm{p} \geq 0,05$ ). Desse modo, além de análises descritivas (frequências, percentagens, médias e 
desvios padrão), utilizou-se também do coeficiente de correlação $r$ de Pearson para verificar relaçôes entre as variáveis deste estudo, e o teste t de Student para verificar diferenças entre as médias obtidas. Com relação aos dados faltantes, a percentagem observada foi inferior a 5\% e optou-se pela exclusão total (listwise) dos questionários que tivessem dados faltantes, não sendo incluídos nas análises realizadas.

Este estudo foi aprovado pelo Comitê de Ética em Pesquisa com Seres Humanos da Universidade Federal de Santa Catarina (UFSC), e todos os procedimentos foram orientados eticamente pelos princípios da Resolução no 196, de 10 de outubro de 1996, do Conselho Nacional de Saúde, e pela Resolução no 16, de 20 de dezembro de 2000, do Conselho Federal de Psicologia.

\section{Instrumentos}

Utilizou-se um questionário autoaplicável, composto por 23 questões (fechadas) subdivididas em 4 setores de interesse:

a) caracterização dos respondentes: características sociodemográficas (6 itens);

b) caracterização de crenças parentais (8 itens);

c) caracterização da mediação parental ( 9 itens). O questionário também continha uma questão aberta no final, na qual os pais poderiam descrever os aspectos positivos e negativos que consideravam associados ao uso da internet pelos filhos.

Para a verificação das crenças e da mediação parental com relação ao uso da internet, duas escalas foram construídas, inspiradas na revisão de literatura. A escala de mediação parental com relação à internet, composta por 9 itens e avaliada por meio de um sistema Likert de 5 pontos (de $1=$ nunca até $5=$ sempre) e baseouse nas três dimensões propostas por Barkin et al. (2006); Livingstone e Helsper (2008); Nikken e Jansz $(2007,2009)$ e também Valcke et al. (2011): mediação ativa (MA; 3 itens), mediação restritiva (MR; 3 itens) e uso acompanhado (UA; 3 itens). Os itens relacionados à MA apresentaram situações de orientação ou discussão crítica dos pais quanto aos jogos, sites ou conteúdos on-line (exemplo de item: "Converso com meus filhos sobre o conteúdo presente nos jogos ou sites que ele visita"); os itens relacionados à MR apresentaram situações de 
aplicação clara de regras para uso da internet, incluindo a proibição de certos jogos ou conteúdos e a restrição do tempo que a criança pode disponibilizar a essa atividade (exemplo de item: "Aplico regras explícitas quanto ao uso de jogos ou sites que meu filho pode acessar on-line"). Os itens relacionados à mediação do tipo "uso acompanhado" (UA) apresentaram situaçôes que incluíam somente a presença do pai ou da mãe durante o período que a criança estava na internet, sem conversa ou orientação sobre a utilização da internet nesse momento (exemplo de item: "Estou por perto quando meu filho utiliza a internet").

A segunda escala, de crenças parentais sobre o uso da internet, foi composta por 8 itens associados a eventuais efeitos ou consequências do uso da internet pelas crianças e avaliada por um sistema do tipo Likert de 5 pontos (de $1=$ discordo totalmente até $5=$ concordo totalmente). Quatro itens dessa escala se referiram a aspectos considerados prejudiciais ou negativos e 4 itens a aspectos benéficos ou positivos ao desenvolvimento da criança. São exemplos desses itens: "Os conteúdos violentos ou agressivos presentes nos jogos on-line podem influenciar negativamente o comportamento das crianças" (crença negativa); "O uso de jogos on-line pode favorecer o desenvolvimento cognitivo de meu filho" (crença positiva).

A averiguação das escalas foi feita por meio de análises relativas à estrutura do instrumento e sua consistência interna, ou seja, por meio da análise fatorial de construto e do Alpha de Cronbach. Assim, os itens específicos de cada escala foram submetidos a uma análise de componentes principais, via método de rotação Varimax com Normalização Kaiser, e os itens com cargas fatoriais iguais ou inferiores a 0,40 foram excluídos. As estruturas propostas para cada escala específica foram confirmadas, e as comunalidades para as duas escalas também foram altas, sendo que as dimensões encontradas para cada escala explicaram de 47,84 a $66,35 \%$ da variância total, obtiveram correlaçôes item-total do fator acima de 0,20 e apresentaram congruência conceitual com os demais itens do mesmo fator.

A análise de consistência interna de ambas as escalas revelou índices aceitáveis para o estudo. O Alpha de Cronbach encontrado para escala total de mediação parental foi de 0,84; sendo que os Alphas de Cronbach para as subescalas de MR; MA e UA foram, respectivamente de 0,63, 0,66 e 0,70. Já o índice de consistência interna total da escala de crenças parentais foi 0,63 , e de suas subescalas, 0,61 (crenças positivas) e 0,60 (crenças negativas). 


\section{Resultados}

Características sociodemográficas da amostra

Os dados referentes a caracterização da amostra podem ser observados na tabela 1. De modo geral, trata-se de uma amostra composta por adultos e adultos jovens de ambos os sexos e, em sua maioria, cursando nível superior no momento do estudo, com um ou dois filhos dentro da faixa etária de interesse, bom nível socioeconômico e cuja carga regular de trabalho diário de oito horas ou mais.

Tabela 1. Características sociodemográficas da amostra $(n=50)$

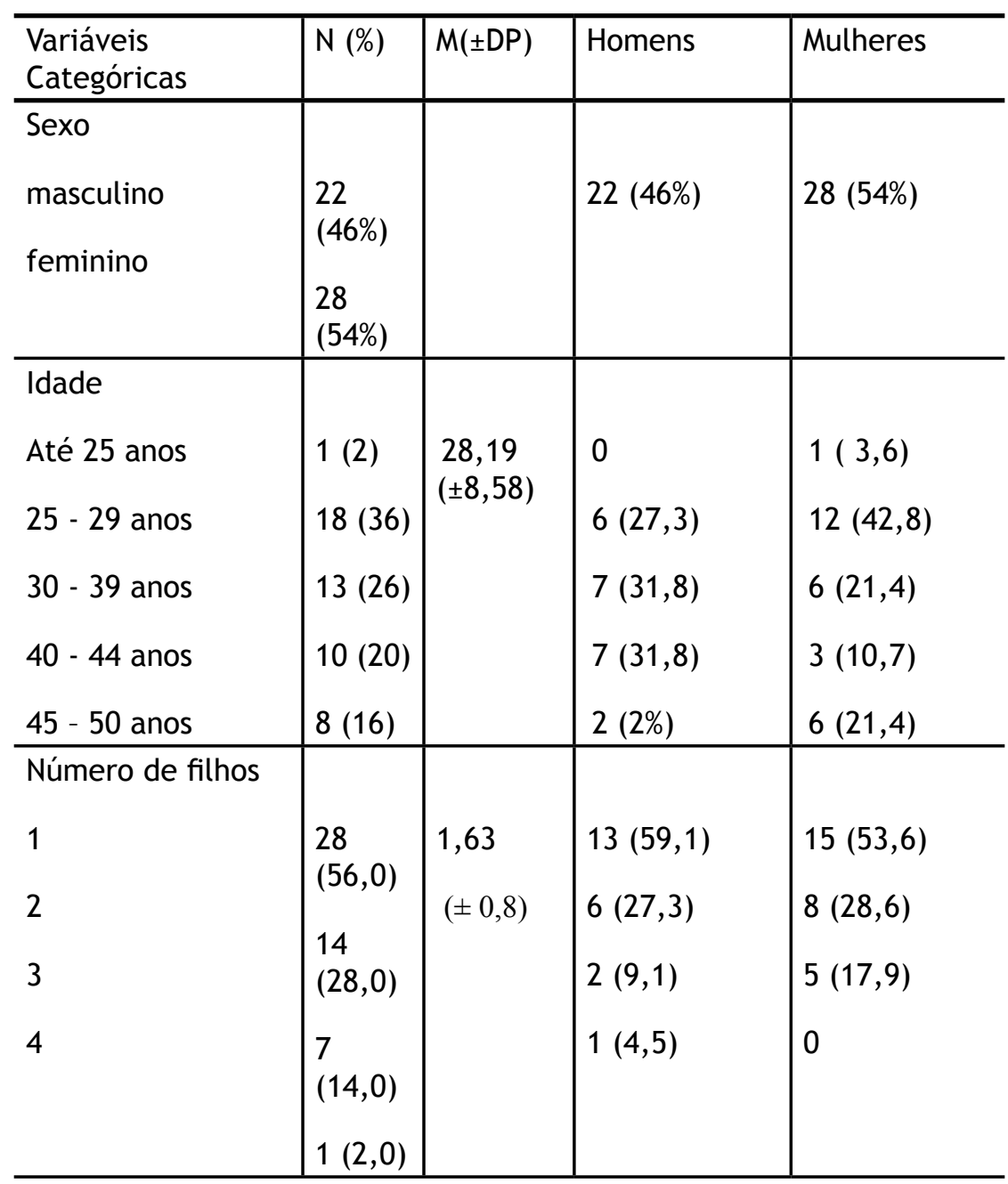




\begin{tabular}{|c|c|c|c|c|}
\hline Nível Escolaridade & & & & \\
\hline $\begin{array}{l}\text { Superior completo } \\
\text { (1) } \\
\text { Superior } \\
\text { incompleto (2) } \\
\text { Pós-graduação (3) }\end{array}$ & $\begin{array}{l}10 \\
(20,0) \\
34 \\
(68,0) \\
6 \\
(12,0) \\
\end{array}$ & $\begin{array}{l}2,00 \\
( \pm 0,70)\end{array}$ & $\begin{array}{l}4(18,2) \\
15(68,2) \\
3(13,6)\end{array}$ & $\begin{array}{l}6(21,4) \\
19(67,8) \\
3(10,7)\end{array}$ \\
\hline $\begin{array}{l}\text { Carga horária de } \\
\text { trabalho diário } \\
4 \text { a } 5 \text { horas (1) } \\
6 \text { horas (2) } \\
7 \text { horas (3) } \\
8 \text { horas ou mais (4) } \\
\text { não respondeu (5) }\end{array}$ & $\begin{array}{l}1(2,0) \\
9(18) \\
7(14) \\
32(64) \\
1(2,0)\end{array}$ & $\begin{array}{l}4,06 \\
( \pm 1,32)\end{array}$ & $\begin{array}{l}0 \\
2(9,1) \\
2(9,1) \\
18(81,8) \\
0\end{array}$ & $\begin{array}{l}1(3,6) \\
7(25) \\
5(17,9) \\
14(50) \\
1(3,6)\end{array}$ \\
\hline $\begin{array}{l}\text { Renda familiar } \\
\text { total (reais) } \\
\text { até } 1.395,00(1) \\
\text { até } 2.325,00(2) \\
\text { até } 3.255,00(3) \\
\text { acima de } 4.185,00 \\
\text { (4) }\end{array}$ & $\begin{array}{l}8 \\
(16,0) \\
13 \\
(26,0) \\
11 \\
(22,0) \\
18 \\
(36,0)\end{array}$ & $\begin{array}{l}3,78 \\
( \pm 1,12)\end{array}$ & $\begin{array}{l}3(13,6) \\
9(40,9) \\
0 \\
10(45,5)\end{array}$ & $\begin{array}{l}5(17,8) \\
4(14,3) \\
11(39,3) \\
8(28,6)\end{array}$ \\
\hline
\end{tabular}

Fonte: elaborado pelos autores.

Crenças parentais quanto ao uso da internet

A escala de crenças parentais contava com 8 frases afirmativas amplamente associadas ao uso da internet pelas crianças: 4 afirmações com aspectos considerados positivos ou benéficos e 4 afirmações com aspectos negativos ou prejudiciais. A análise descritiva dos resultados em relação às opções de 
resposta "sempre" e "quase sempre" a essas afirmativas permitiu identificar que entre as crenças positivas, a grande maioria dos pais (74\%) acreditam na aquisição de habilidades e benefícios cognitivos e de desenvolvimento, e entre as principais crenças negativas investigadas, os pais (73\%) acreditam no aumento da agressividade. Conforme observado nas respostas dadas à única pergunta aberta do instrumento, as crenças positivas fazem maior alusão ao "aumento na inteligência" e "agilidade mental",4 e as negativas, às consequências relacionadas à exposição da criança a conteúdos violentos e, ou, sexuais.

A análise estatísticas dos resultados por meio do teste t de Student também revelou diferença estatística $[\mathrm{t}(48)=39,52 ; \mathrm{p}=0,000]$ entre as médias das crenças positivas $(\mathrm{M}=30,58 ; \mathrm{DP}=4,44)$ e negativas $(\mathrm{M}=18,84 ; \mathrm{DP}=3,37)$, o que significa dizer que os pais responderam "sempre" e "quase sempre" a um número maior de afirmativas relacionadas aos aspectos positivos ou benéficos, revelando otimismo com relação aos efeitos do uso da internet pelas crianças.

Mediação parental com relação ao uso da internet pelas crianças

Este estudo considerou o uso de três mediações possíveis para o gerenciamento do uso da internet pelas crianças (MA, MR e UA). Embora não se tenha detectado diferença estatística entre as médias, observou-se que a MR foi o tipo de mediação que obteve a menor média $(M=9,68 ; \mathrm{DP}=2,89)$, bem como apresentou menor frequência de uso (30\%), o que nos permite inferir que os pais preferem conversar e orientar (MA; $M=11,38$ e $\mathrm{DP}=2,60)$ ou acompanhar (UA; $\mathrm{M}=11,10$ e $\mathrm{DP}=2,27$ ) os filhos em suas atividades na internet, visto que esses tipos de mediação obtiveram médias e percentuais similares entre si, e são mais utilizadas pelos pais ( $48 \%$ e $44 \%$, respectivamente) que a MR.

A análise de correlação feita por meio do $r$ de Pearson (tabela 2) não encontrou relações entre as crenças (positivas e negativas) e os tipos de mediação utilizadas pelos pais, indicando que, possivelmente, outros fatores não contemplados neste estudo podem ter maior influência na escolha da maneira como gerenciam o contato dos filhos com a internet.

Conforme também se observa na tabela 2 , entre os tipos de mediação, há uma correlação positiva moderada entre a MA e a MR $(r=0,62 ; p<0,001)$ e entre a MA e UA $(r=0,64 ; \mathrm{p}<0,001)$, indicando que os pais que mais conversam e orientam (MA) também são os que mais acompanham (UA) e mais regulam (MR) o uso da internet pelos filhos. A análise dos dados também revelou uma fraca associação positiva $(r=0,27 ; \mathrm{p}<0,05)$ entre a mediação UA e o sexo do respondente. A comparação das médias realizadas por meio do teste t-Student para amostras

4 Termo utilizado neste estudo para agrupar as respostas dos pais, feitas primordialmente com expressóes do tipo "ligeiro nas ideias", "rápido de pensamento", "raciocínio melhorado", "esperto", "ladino", etc. 
independentes indicou diferença significante $[\mathrm{t}(48)=1,96 ; \mathrm{p}=0,000]$ entre o uso da mediação UA por pais e mães, sendo que elas $(\mathrm{M}=11,67 ; \mathrm{DP}=2,18)$ fazem maior uso desse tipo de mediação que eles $(M=10,43$; $D P=2,23)$. Investigando esse resultado por meio de uma análise mais detalhada, verificou-se fraca associação negativa entre as variáveis carga de trabalho e sexo do respondente $(\mathrm{r}=$ $0,37 ; \mathrm{p}<0,05)$, e o teste $\mathrm{t}$-Student revelou diferença significativa $[\mathrm{t}(48)=2,43$; $\mathrm{p}=0,04]$ entre as médias da variável carga de trabalho de pais $(\mathrm{M}=4,55 ; \mathrm{DP}=1,01)$ quando comparada à das mães $(\mathrm{M}=3,65 ; \mathrm{DP}=1,0)$; o que pode indicar que elas passam mais tempo com os filhos devido a carga de trabalho menor.

Tabela 2. Apresentação das principais correlações entre as variáveis do estudo

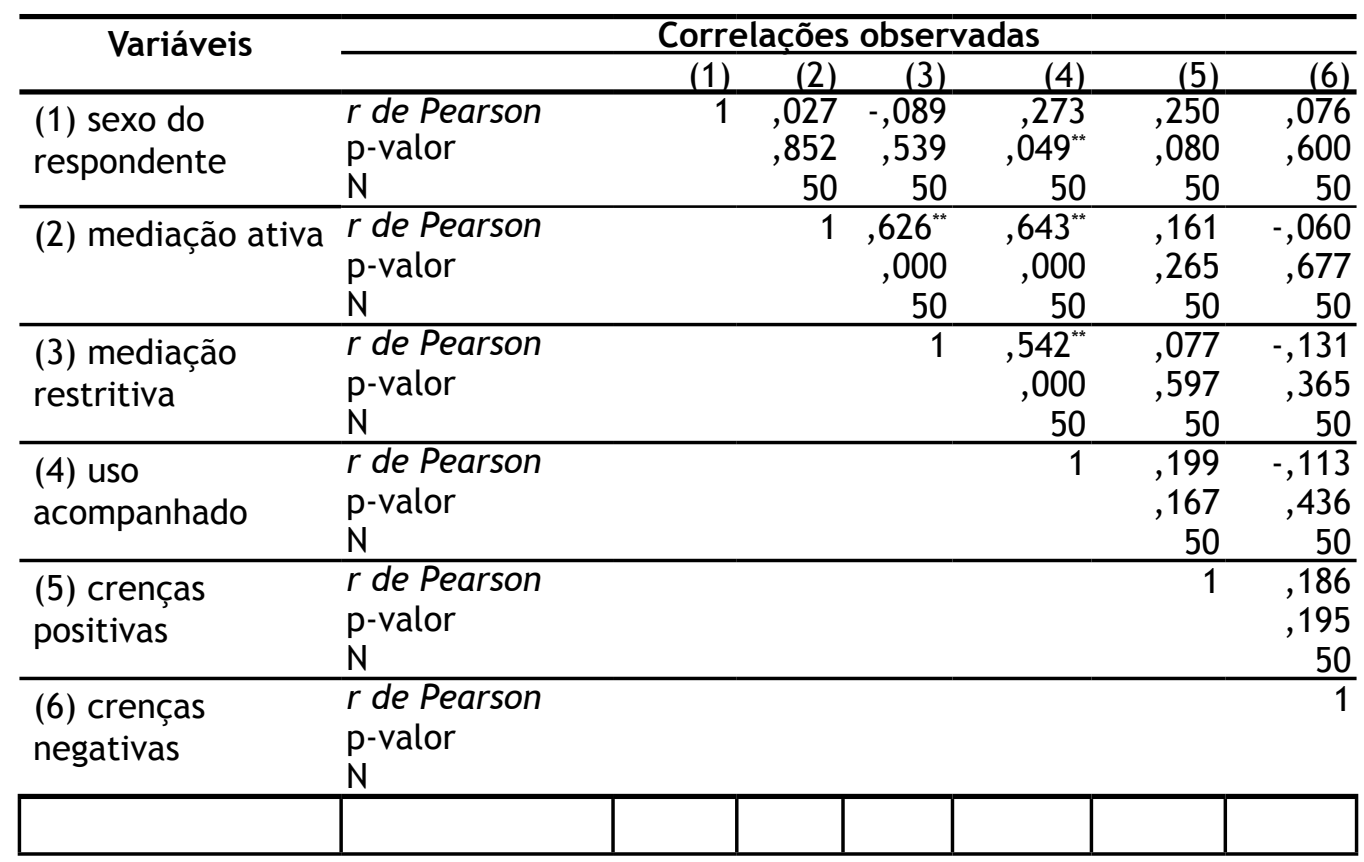

Fonte: elaborado pelos autores.

\section{Discussão}

Dentro do contexto de desenvolvimento humano, ressalta-se a importância e o papel dos pais como agentes preditores de vários aspectos do comportamento das crianças e adolescentes. Conhecer os aspectos que influenciam o tipo de mediação em relação ao uso da internet pelas crianças pode contribuir para melhor orientá-los nesse processo, de modo a potencializar os efeitos benéficos e desejáveis desse recurso para o desenvolvimento infantil.

Pela análise de dados, identificou-se que as principais crenças positivas apontadas pela grande maioria dos pais referem-se à influência sobre a vida 
escolar da criança e a aquisição de habilidades consideradas necessárias para a vida adulta. Em contraponto, as principais crenças negativas indicadas referem-se à influência negativa decorrente da exposição a conteúdos impróprios bem como à possível estimulação de comportamentos agressivos. Apesar de conscientes dos riscos e benefícios, nossos resultados sugerem certo otimismo por parte dos pais com relação aos efeitos do uso da internet pelas crianças.

Nesse sentido, nossos dados parecem admitir as considerações de Livingstone e Bober (2006), Livingstone e Haddon (2008), e também as de Çankaya e Odabasi (2009), que apontam para uma visão ainda ambivalente dos pais quanto aos efeitos da internet, principalmente diante das diversas influências positivas e negativas amplamente noticiadas para o uso das crianças. E também as consideraçôes de Ortiz, Green e Lim (2011), cujos pais veem a relação entre os filhos e a internet quase que exclusivamente de maneira positiva, sobretudo no que se refere à vida acadêmica e possibilidade futura de ser bem-sucedido profissionalmente.

Referente aos tipos de mediação parental para o uso da internet pelas crianças, muito embora as mães se utilizem mais da mediação UA que os pais, os dados indicaram que a maioria de nossa amostra prefere conversar e orientar ou acompanhar os filhos em suas atividades na internet, mas também aplica algumas regras e restrições para o uso, caracterizando um tipo de mediação mista (MA, MR, UA). Nossos resultados foram similares aos obtidos por Barkin et al. (2006) em uma amostra de pais do Canadá e de Porto Rico, e aos que Livingstone e Bober (2006) encontraram na amostra de pais do Reino Unido, onde a maioria dos pais prefere o uso de múltiplas estratégias de mediação (UA, MA, MR). A preferência geral dos pais por uma mediação do tipo mista pode ser indicativa de que estes ainda se encontram divididos quanto ao melhor modo de fazê-lo, sugerindo que esse ainda é um campo em construção no seio das famílias. No entanto a preferência pela mediação ativa e pelo uso acompanhado observados em nosso estudo também pode ser reflexo do grau de escolaridade e nível socioeconômico, pois, como pontuado por Almeida et al. (2011), pais com melhores níveis educacionais e socioeconômicos adotam uma abordagem mais próxima para guiar o uso da internet de seus filhos, como acompanhamento, orientação e conversas. Quanto ao maior uso das mães de nossa amostra pela mediação do tipo uso acompanhado, talvez se deva por estarem mais presentes em casa quando os filhos usam a internet (devido à carga de trabalho menor que a observada para os pais), ou ainda, como menciona Morgado (2000), porque culturalmente se espera que a mulher ocupe o principal espaço de educadora dos filhos, que converse mais com eles e que tenha maior preocupação com os problemas familiares. 
Em relação ao contexto físico e social da vida diária da criança, como acesso ao computador e internet, bem como o conjunto de regras utilizadas para o uso ou manipulação desses artefatos (tempo, atividades permitidas, etc.), os pais de nossa amostra parecem favorecer o contato das crianças com as chamadas tecnologias digitais por meio de um ambiente midiático rico e de pouco controle sistemático. Nesse sentido, nossos dados acompanham uma tendência mundial, reflexo de uma cultura mais ampla, na qual a infância de hoje está relacionada diretamente ao franco acesso e uso de tecnologia e ao otimismo diante das decorrências desse uso, especialmente no âmbito acadêmico e profissional. Pode-se dizer que os pais parecem envaidecidos com a facilidade apresentada pelas crianças para lidar com tais artefatos, e os disponibilizam cada vez mais cedo aos filhos, sendo comum observarmos crianças muito novas, mesmo bebês, manusearem tablets e computadores. Assim, conforme crescem, torna-se natural e fácil para as crianças o uso de outros recursos mais sofisticados (que por vezes são até complicados para o entendimento dos pais, que tendem muitas vezes a superestimar as habilidades e competências dos filhos diante de tais recursos). A esse respeito também se destaca a acentuada presença da chamada "socialização reversa" na relação pais-filhos-internet, ou seja, o fato de as crianças usarem naturalmente e sem dificuldade vários recursos e aplicativos acaba auxiliando a introduzir (ou aprofundar) os pais nesse universo de tecnologia e a reforçar o estereótipo autossuficiente das crianças nesse âmbito.

Quanto à pergunta de pesquisa formulada para este estudo, verificou-se que as crenças dos pais sobre os eventuais efeitos do uso da internet não alteram a sua gestão, ou seja, não alteram o tipo de mediação utilizada para guiar o uso dos filhos na internet. Tal resultado foi uma surpresa, pois se esperava encontrar, entre os pais com crenças mais negativas, alguma associação a maiores níveis de mediação restritiva e, entre os pais com crenças mais positivas, menores níveis de controle ou regras (o que não foi observado). Entretanto os resultados obtidos neste estudo precisam ser relativizados, uma vez que se trata de um estudo piloto, o tamanho da amostra é limitado, e as escalas usadas ainda não foram validadas.

Acredita-se que o estudo proposto tenha sua validade por instigar a discussão acerca da importância dos aspectos que influenciam o comportamento dos pais na orientação de seus filhos junto à internet, especialmente, porque o uso de tal recurso e as estratégias parentais empregadas têm implicações associadas ao comportamento e desenvolvimento infantil. 


\section{Conclusões}

Em nosso estudo, apesar de se mostrarem divididos quanto aos benefícios e malefícios oportunizados pelo uso da internet, os pais assumem uma postura mais próxima para guiar o comportamento dos filhos quanto a esse uso, preferindo conversar e orientar a criança, o que é considerado pela literatura como o mais adequado para auxiliar as crianças a desenvolverem competências para o uso responsável e seguro da internet. Contudo essa conclusão deve ser cautelosa por conta das limitaçóes do estudo, por exemplo, o tamanho reduzido da amostra e suas características, a ausência de informações do ponto de vista das crianças, a não aplicação piloto do instrumento e, ainda, a não validação das escalas de crenças e de mediação utilizadas (embora tenham apresentado consistência interna aceitável, sua estrutura fatorial não chegou a ser verificada devido ao número reduzido de respondentes). Como sugestões para trabalhos futuros, recomenda-se além da ampliação da amostra, averiguar se existem diferenças entre o comportamento dos pais no que tange a aspectos gerais da criação dos filhos e a orientação dos filhos para o uso da internet, bem como, verificar se outros aspectos como o próprio uso da internet pelos pais, as preocupaçóes quanto a esse uso pelas crianças, o gênero ou idade dos pais e filhos, etc., influenciam tais resultados. Outra sugestão ainda, seria a inclusão das crianças nas investigações.

Por fim, destaca-se que as peculiaridades existentes nas relações envolvendo o gerenciamento da internet no ambiente familiar é um campo fértil de estudo no âmbito da parentalidade e que ainda carece de maiores investigações especialmente da Psicologia.

\section{Referências}

Almeida, A. N., Alves, N. A. \& Delicado, A. (2011). As crianças e a internet em Portugal: perfis de uso. Sociologia, Problemas e Práticas, 65, 9-30.

Almeida, A. N., Alves, N. A., Delicado, A. \& Carvalho, T. (2011). Children and digital diversity: from "unguided rookies" to "self-reliant cybernauts". Childhood, 19 (2), 1-16.

Alves, L. R. G. (2005). Game over: jogos eletrônicos e violência. São Paulo: Futura.

Aslanidou, S. \& Menexes, G. (2008). Youth and the internet: uses and practices in the home. Computers \& Education, 51 (3), 1375-1391. 
Austin, E. W. (1993). Exploring the effects of active parental mediation of television content. Journal of Broadcasting \& Electronic Media, 37, 147-158.

Barkin, S., I., E., Richardson, I., Klinepeter, S., Finch, S. \& Krcmar, M. (2006). Parental media mediation styles for children aged 2 to 11 years. Archives of pediatrics \& adolescent medicine, 160 (4), 395-401.

Çankayaa, S. \& Odaba 1, H. F. (2009). Parental controls on children's computer and Internet use. Procedia - Social and Behavioral Sciences, 1 (1), 1105-1109.

Chang, M. K. \& Law, S. P. M. (2008). Factor structure for young's internet addiction test: a confirmatory study. Computers in Human Behavior, 24, 2597-2619

Comitê Gestor da Internet no Brasil (2011). Relatórios de pesquisas: TIC Domicílios e Empresas 2010 e TIC Educação 2010. Recuperado a partir de http://www.cgi.br/.

Eastin, M., Greenberg, B. S. \& Hofschire, L. (2006). Parenting the internet. Journal of Communication, 56, 486-504.

Fujioka, Y. \& Austin, E. W. (2002). The relationship of family communication patterns to parental mediation styles. Communication Research, 29 (6), 642665.

Gentile, D. A. \& Stone, W. (2005). Violent video game effects on children and adolescents: a review of the literature. Minerva Pediatrica, 57, 337-358.

Jackson, L. A., Eye, A. von, Witt, E. A., Zhao, Y. \& Fitzgerald, H. E. (2011). A longitudinal study of the effects of internet use and video game playing on academic performance and the roles of gender, race and income in these relationships. Computers in Human Behavior, 27, 228-239.

Kuhlemeier, H. \& Hemler, B. (2007). The impact of computer use at home on student's internet skills. Computer \& Education, 49 (2), 460-480.

Kutner, O., Olson, C. K., Warner, C. K. \& Herzog, S. M. (2008). Parents' and son's perspectives on videogame play. Journal of Adolescent Research, 23 (1), 76-96.

Lee, S. J. \& Chae, Y. G. (2007). Children's internet use in a family context: influence on family relationships and parental mediation. Cyberpsychology o 
Behavior: the impact of the Internet, multimedia and virtual reality on behavior and society, 10 (5), 640-644.

Livingstone, S. \& Bober, M. (2006). Regulating the internet at home: contrasting the perspectives of children and parents in digital generations: children, young people, and the new media. In D. Buckingham \& R. Willett. Digital generations: chidren, young people and new media. (pp. 93-113). Mahwah: Lawrence Erlbaum.

Livingstone, S. \& Haddon, L. (2008). Risky experiences for children online: charting European research on children and the internet. Children \& Society, $22(4), 314-323$.

Livingstone, S., Haddon, L., Görzig, A. \& Ólafsson, K. (2011). Risks and safety on the internet: the perspective of European children. Full findings. London: LSE, EU Kids Online. Recuperado a partir de http://www.lse.ac.uk/ media\%40lse/research/EUKidsOnline/EU\%20Kids\%20II\%20(2009-11)/ EUKidsOnlineIIReports/D4FullFindings.pdf

Livingstone, S. \& Helsper, E. J. (2008). Parental mediation of children's internet use. Journal of Broadcasting \& Electronic Media, 52 (4), 581-599.

Lwin, M. O., Stanaland, A. \& Miyazaki, A. (2008). Protecting children's privacy online: how parental mediation strategies affect website safeguard effectiveness. Journal of Retailing, 84, 205-217.

Mendes, N. M. S. (2011). O impacto do cibersexo na qualidade de vida e na saúde mental. (Dissertação de mestrado). Universidade Lusófona de Humanidades e Tecnologias, Aconselhamento e Psicoterapia, Lisboa.

Mesch, G. S. (2009). Parental mediation, online activities and cyberbullying. Cyber Psychology \& Behavior, 12 (4), 387-393.

Mondin, E. M. C. (2008). Práticas educativas parentais e seus efeitos na criação dos filhos. Psicologia Argumento, 26 (54), 233-244.

Morgado, R. (2000). Família(s) e relações de gênero. Praia Vermelha: estudos de política e teoria social, 1 (1), 190-215.

Morrison C. M. \& Gore, H. (2010). The relationship between excessive internet use and depression: a questionnaire-based study of 1,319 young people and adults. Psychopathology, 43, 121-126. 
Mungunba, M. C., Valdés, M. T. M., Matos, V. C. \& Silva, C. A. B. (2003). Jogos eletrônicos: apreensão de estratégias de aprendizagem. Revista Brasileira em Promoção da Saúde, 16 (1-2), 39-48.

Nathanson, A. (2001). Parent and child perspectives on the presence and meaning of parental television mediation. Journal of Broadcasting \& Electronic Media, 45, 201-220.

Nathanson, A. (2002). The unintended effects of parental mediation of television on adolescents. Media Psychology, 4, 207-230.

Nikken, P. \& Jansz, J. (2007) Playing restricted videogames: relations with game ratings and parental mediation. Journal of Children and Media, 1 (1), 227-243.

Nikken, P. \& Jansz, J. (2009). Playing restrict videogames: relations with game ratings and parental mediation. Journal of Children and Media, 1 (3), 227243.

O'Keeffe, G. S. (2010). CyberSafe: protecting and empowering kids in the digital world of texting, gaming, and social media. Washington: American Academy of Pediatrics.

Ortiz, R. W., Green, T. \& Lim, H. (2011). Families and home computer use: exploring parent perceptions of the importance of current technology. Urban Education, 46 (2), 202-215.

Pasquier, D. (2001). Media at home: domestic inter-actions and regulation. In S. Livingstone \& M. Bovill (Eds), Children and their changing media environment: a European comparative study. (pp. 161-177). Mahwah: Lawrence Erlbaum.

Pijpers, R. \& Pardoen, J. (2009). Next level: dossier on online games for children. Den Haag: Stichting Mijn Kind.

Ponte, C. \& Vieira, N. (2008). Crianças e internet, riscos e oportunidades: um desafio para a agenda de pesquisa nacional. In Moisés de Lemos Martins \& Manuel Pinto (Orgs.), Comunicação e Cidadania - Atas do $5^{\circ}$ Congresso da Associação Portuguesa de Ciências da Comunicação. (pp. 2732-2741). Braga: Centro de Estudos de Comunicação e Sociedade (Universidade do Minho). Recuperado a partir de: revistacomsoc.pt/index.php/5sopcom/article/ download/236/255. 
Rodríguez, G. M. P. \& Morales, R. V. (2007). La adicción a internet en adolescentes, se asocia con síntomas psiquiátricos como, TDAH, depresión y hostilidad. Evid. Pediatr., 3 (104). Recuperado a partir de: http://archivos.evidenciasenpediatria.es/files/41-10599-RUTA/La\%20 adicci\%C3\%B3n\%20a\%20internet $\% 20$ en\%20adolescentes.pdf

Rosen, L. D., Cheever, N. A. \& Carrier L. M. (2008). The association of parenting style and child age with parental limit setting and adolescent MySpace behavior. Journal of Applied Developmental Psychology, 29, 459-471.

Subrahmanyam, K., Kraut, R., Greenfield, P. \& Gross, E. (2000). The impact of home computer use on children's activities and development. Children and Computer Technology, 10 (2), 123-144.

Swing, E. L., Gentile, D. A. \& Anderson, C. A. (2008). Violent video games: learning processes and outcomes. In R. E. Ferdig (Ed.), Handbook of research on effective electronic gaming in education. (pp. 876-892). Hershey: Information Science Reference.

Tabone, S. \& Messina, L. (2010). Personal uses of internet and perceptions of parental mediation: a research with children 10 and 11 years old. Children, 2, 2077-2082.

Tripp, L. M. (2010). The computer is not for you to be looking around, it is for schoolwork': challenges for digital inclusion as Latino immigrant families negotiate children's access to the internet. New Media \& Society, 13 (4), $552-567$.

Turkle, S. (1995). Life on the screen: identity in the age of the internet. New York: Simon and Schuster.

Valcke, M., Bonte, S., De Wever, B. \& Rots, I. (2010). Internet parenting styles and the impact on Internet use of primary school children. Computers \& Education, 55 (2), 54-464.

Valcke, M., De Wever, B., Van Keer, H. \& Schellens, T. (2011). Long-term study of safe Internet use of young children. Computers \& Education, 57 (1), 1292-1305.

Valkenburg, P. M., Krcmar, M., Peeters, A. L. \& Marseille, N. M. (1999). Developing a scale to assess three styles of television mediation: "Instructive mediation", "restrictive mediation", and "social coviewing". Journal of Broadcasting \& Electronic Media, 43, 52-66. 
Warren, R. (2005). Parental mediation of children's television viewing in lowincome families. Journal of Communication, 55, 847-863.

Weinberger, N., Anderson, T. \& Schumacher, P. (2009). Young children's access and use of computers in family child care and child care centers. Computers in Human Behavior, 25, 183-190.

Wilson, K. A., Bedwell, W. L., Lazzara, E. H., Salas, E., Burke, C. S., Estock, E. L., Conkey, C. (2009). Relationships between game attributes and learning outcomes. Review and Research Proposals Simulation Gaming, 40 (2), 217-266. 